\title{
Risk Factors for Lower Extremity Fatigue Among Assembly Plant Workers
}

\author{
Nancy Gell, PT, MPH, ${ }^{1}$ Robert A. Werner, MD, MS, ${ }^{1,2,3 *}$ Anne Hartigan, MD, ${ }^{1}$ \\ Neal Wiggermann, $\mathrm{MS}^{3}$ and $\mathrm{W}$. Monroe Keyserling, $\mathrm{PhD}^{3}$
}

Background Work-related fatigue of the lower extremities is a known cause of lost productivity and significant employer costs. Common workplace solutions to reduce fatigue levels include anti-fatigue matting, shoe orthoses, or sit/stand work stations. However, assessment of these anti-fatigue measures within the workplace has been limited. Methods This was a cross sectional study in an automotive assembly plant on employees with at least 6 months tenure. Subject data were collected via questionnaires including Likert-scale questions to define fatigue severity. Jobs were evaluated for lower extremity ergonomic exposures via videotaping, pedometers, interviews, and industrial engineering records.

Results Lower extremity fatigue at the end of the work day was associated with a higher prevalence of smoking, rheumatoid arthritis, job dissatisfaction, use of shoes with firmer outsoles, and increased time on the job spent standing or walking. Supervisor support and increased time spent on carpet were protective. Lower extremity fatigue that interfered with activities outside of work had additional risk factors including higher BMI, priordiagnosis of osteoarthritis, and increased hours per week spent working.

Conclusions While these results identify carpet as being protective against lower extremity fatigue, no similar relationship was identified for anti-fatigue mats. No adverse relationship was found between hard surfaces such as concrete and lower extremity fatigue. Given the high costs associated with work-related fatigue, future areas for potential intervention include smoking cessation, specific shoe recommendations, and enhancing psychosocial aspects of work such as supervisor support. Am. J. Ind. Med. 54:216-223, 2011. ๑ 2010 Wiley-Liss, Inc.

KEY WORDS: fatigue; lower extremity; work-related

${ }^{1}$ Department of Physical Medicine and Rehabilitation, University of Michigan Health System, Ann Arbor, Michigan

${ }^{2}$ Veteran Affairs Health System, Ann Arbor, Michigan

${ }^{3}$ Center for Ergonomics, Industrial and Operations Engineering, University of Michigan, Ann Arbor, Michigan

Contract grant sponsor: United Auto Workers (UAW) and General Motors (GM) National Joint Committee on Health and Safety.

${ }^{*}$ Correspondence to: Robert A. Werner, Chief, PM\&R, Ann Arbor VAMC, 2215 Fuller Rd (117), Ann Arbor, MI 48105. E-mail: rawerner@umich.edu

Accepted 11 September 2010

DOI 10.1002/ajim.20918. Published online 28 October 2010 in Wiley Online Library (wileyonlinelibrary.com).

\section{INTRODUCTION}

Work-related fatigue has been examined in numerous forms including generalized fatigue, lower extremity fatigue, and fatigue in combination with discomfort and pain. Varying definitions of fatigue have been used and there is little consistency in how both generalized and lower extremity fatigue have been assessed in different studies. While population based studies have looked for multifactorial risks for generalized work related fatigue, studies examining lower extremity fatigue have focused on comparisons between different standing surfaces and shoe types and 
their effects on lower extremity fatigue in a laboratory setting.

Prior studies on general fatigue in the working population have found a prevalence ranging from $7 \%$ to 45\% [Chen, 1986; Lewis and Wessely, 1992; Ricci et al., 2007]. A recent study by Ricci et al. [2007] found a prevalence of fatigue of $38.9 \%$ with an estimated cost of over $\$ 1$ billion related to lost productivity. The prevalence of work-related lower extremity fatigue has not been documented. However, studies have shown an association between lower extremity fatigue and standing work [Redfern and Chaffin, 1995; Redfern and Cham, 2000]. Common workplace solutions to reduce fatigue levels include antifatigue matting, shoe orthoses, or sit/stand work stations. However, assessment of the effects of these anti-fatigue measures within the workplace has been limited.

The prevalence of muscle fatigue is difficult to evaluate since fatigue is often associated with other health conditions and there is no well-defined diagnostic tool to identify this condition [De Vries et al., 2003]. While some laboratory studies [Kim et al., 1994; Cham and Redfern, 2001], used physiological measures (surface EMG recordings, skin temperature, volumetric measurements) to correlate physiological changes with subjective ratings of fatigue, the challenge for population-based studies is how to measure lower extremity fatigue in a meaningful way. Subjective measures, typically using a Likert scale, have been the most common means of assessing general and lower extremity fatigue in population-based studies. In three studies [Redfern and Chaffin, 1995; King, 2002; Orlando and King, 2004] lower extremity fatigue was rated on a 5 point scale with the anchors "not tired" and "very tired." Cham and Redfern [2001] asked participants to rate overall leg fatigue on a 10 point Borg scale. Each of these studies asked subjects to rate "discomfort" in addition to the fatigue ratings. All of the aforementioned studies asked subjects to provide fatigue ratings for specific time periods as defined by each study.

The purpose of this study was to examine lower extremity fatigue and its relationship to ergonomic, medical and demographic factors among industrial workers. Fatigue was defined as a feeling of tiredness, weariness, or exhaustion in the legs and rated using a 5 point Likert scale with verbal anchors. This was modified for the lower extremities based on a general definition of fatigue [MedlinePlus, 2007]. In contrast to previous studies, this study aimed to define the intensity and impact of fatigue by differentiating between fatigue at the beginning or end of the work day, and fatigue that interferes with both work and non-work activities. Similarly to previous studies, subjects were asked to rate pain perception separately from fatigue. Additionally, subjects were asked to relate their lower extremity fatigue to a typical work day as opposed to a specific point in time. The goal was to determine the relative contributions of different work activities (percent time spent standing, walking, or sitting), floor surface characteristics, weight, body mass index (BMI), age, foot biomechanics, hours worked per week and other demographic and medical history factors to the prevalence of lower extremity fatigue among workers in an automobile engine assembly plant.

\section{METHODS}

This was a cross-sectional study conducted at an automotive engine manufacturing plant in the Northern United States. All salaried and hourly plant employees including those from assembly, machining, skilled trades, and administration were eligible to participate. Participation included completing a symptom questionnaire, undergoing a physical examination and work exposure assessment based on a job analysis that focused on lower body posture. Data collection for the symptom questionnaire and physical examination portions of the study was performed during non-work times, including breaks and before- or after-work hours. The work exposure assessments were conducted during regular work hours. All subjects signed a consent form approved by the Institutional Review Board at the University of Michigan. Workers who agreed to participate in the study were given a t-shirt and those who completed all aspects of the study received a $\$ 25$ participation incentive.

Participants completed a lower extremity symptom questionnaire which included a body diagram for pain, surgical history, medications history, and documented hours worked in the prior week. Demographics such as age, height, weight, and past medical history for both generalized and lower extremity disorders were included in the questionnaire. Smoking history and exercise were self-reported. Additionally, subjects completed questions focused on regional lower extremity pain that occurred within the last year and that lasted more than 1 week or occurred more than three times. The symptom questionnaire included a section based on the Job Content Questionnaire by Karasek et al. [1998] including scales to assess job satisfaction, supervisor support, and decision authority. The score for supervisor support is created from four questions with a potential score range of 4-16. Job dissatisfaction has a score range of $0-10$ based on a construction from five questions. The score for decision authority is based on a formula to combine responses to three questions with a score range of 12-48.

Participants were asked to rate their level of agreement with statements such as "At the end of a typical work day my legs and/or feet feel fatigued" and "fatigue in my legs and/or feet from work interferes with activities outside of work," in addition to questions about lower extremity fatigue at the beginning and end of a typical work week using a 5 point Likert type scale ranging from "strongly disagree" to "strongly agree." For the purpose of this study, analysis focused on "fatigue at the end of a typical work day" and "fatigue that interferes with activities outside of work." For 
statistical analysis the responses were collapsed to create a dichotomous variable for fatigue with "strongly agree" and "agree" assigned to "yes" and "strongly disagree," "disagree" and "neither agree not disgaree" assigned to "no." For the purpose of differentiating between treatment for fatigue and treatment for pain and/or discomfort, subjects were asked about treatments utilized for each in separate questions. Subjects were asked if they ever tried to treat their fatigue, types of treatment received, and level of improvement in fatigue after treatment. Two questions utilized in the 1998 Quebec Health and Social Survey assessing freedom to sit or stand at will were included in this survey for comparison [Messing et al., 2008].

Subjects underwent a focused physical examination of the lower extremities to evaluate the hip, knee, and ankle joints as well as standing foot alignment, posture, and gait analysis. Details of the physical examination are described by Werner et al. [2010], In addition to the physical examination, a mechanical evaluation of the shoe was also performed. The hardness of the inner and outer sole of subjects' shoes was measured using a type C durometer (Pacific Transducer Corp, Los Angeles, CA). For both the outer and the inner surface, three locations were tested and the highest measurement was recorded. For the purposes of analysis the durometer recordings were categorized as "low," "medium," and "high." The distribution of the recordings showed a natural clustering with the majority of readings (62\%) lying between 18 and 32 (range 4-92). Based on this distribution it was decided to categorize the durometer readings into low, medium, and high categories with the low category having durometer recordings less than 18 and the high category with reading greater than 32 .

The evaluation of lower body posture/activity and floor surface was performed on volunteers as they performed their regular jobs. All jobs were assessed and ergonomic exposures (i.e., time spent in specific lower body postures, and time spent on a particular surface) were calculated as percentages of a typical work day. The primary mode of exposure assessment was video analysis with additional data collected via pedometer analysis and interviews. Detailed information on the methods used for video analysis of assembly jobs can be found elsewhere [Keyserling et al., 2010]. For jobs where the worker was not confined to a specific work area (i.e., skilled trades, drivers, machinists, and administrative staff), video recordings, interviews, engineering records, and pedometer recordings were used to collect lower body exposure data.

\section{Statistical Analysis}

A univariate analysis using $t$-tests (for continuous variables) or Chi-square tests (for categorical variables) was performed. Subjects with and without lower extremity fatigue at the end of the work day were compared for baseline demographics, medical history, ergonomic exposures, psychosocial factors, discomfort ratings, and regional medical outcomes. Logistic regression was performed using dichotomized responses for "lower extremity fatigue at the end of the work day" and "lower extremity fatigue that interferes with activity outside of work" as the dependent variables and demographic, medical history and ergonomic risk factors as the independent variables to create predictive models. The predictive model was created using backwards stepwise regression with a $P>0.2$ used as a cutoff for eliminating independent variables. We controlled for age, gender and BMI by forcing these variables in the model.

\section{RESULTS}

A total of 407 completed the study, $61 \%$ of the total number of eligible employees. The average age in years of the cohort was $48.4 \pm 10.3$ (mean \pm standard deviation) with $73 \%$ having spent more than 20 years at the company. The cohort was $76 \%$ men with a mean BMI of $29.4 \pm 5.3$. Two hundred seventy-seven subjects (68\%) reported experiencing lower extremity fatigue at the end of a typical work day. Sixty subjects (15\%) disagreed or strongly disagreed with the statement "At the end of a typical work day my legs and/or feet feel fatigued" and 66 subjects (16\%) neither agreed or disagreed with the statement. One hundred thirty eight subjects (34\%) agreed or strongly agreed with the statement "fatigue in my legs and/or feet from work, interferes with activities outside of work," and 153 subjects (38\%) disagreed or strongly disagreed. One hundred fifteen subjects $(28 \%)$ neither agreed or disagreed with the statement. Twenty percent of the study cohort undertook treatment for lower extremity fatigue which included support hose, shoe inserts, massage, and whirlpool.

A univariate comparison is presented in Table I between workers who reported lower extremity fatigue at the end of the day and those who did not. There was no difference between groups for age, BMI or gender distribution; however those without fatigue had fewer years working in auto manufacturing. Subjects with lower extremity fatigue were more likely to have a history of smoking and rheumatoid arthritis. Pain in the lower extremities was strongly associated with fatigue. Subjects reporting lower extremity fatigue had less job decision making authority, less supervisor support, greater job dissatisfaction, and less co-worker support. Subjects who spent more time standing or walking on carpet had lower prevalence of fatigue. Among subjects who sat for part of the work day, those who reported having an option to get up at will, as compared to those indicating they did not have the ability to get up or were only able to get up once in a while, were significantly less likely to report fatigue at the end of the day. Differences in footwear were significant between the two groups. Subjects using shoe inserts and 
TABLE I. Demographic, Medical History, Ergonomic Factors in Subjects With and Without Fatigue at the End of the Work Day ( $N=307)$

\begin{tabular}{|c|c|c|c|}
\hline Demographic factor & $\begin{array}{l}\text { Subjects with fatigue at the end of } \\
\text { the day } \mathrm{N}=\mathbf{2 7 7}(68 \%)\end{array}$ & $\begin{array}{l}\text { Subjects without fatigue at the } \\
\text { end of the day } N=130(32 \%)\end{array}$ & $P$-Value \\
\hline $\mathrm{Age}^{\mathrm{a}}$ & $49.0(9.5)$ & $47.2(11.8)$ & 0.11 \\
\hline Body mass index & $29.7(5.5)$ & $28.8(5.0)$ & 0.12 \\
\hline Gender ( $\%$ female) & 26 & 19 & 0.16 \\
\hline Weight & $202.3(42.3)$ & $199.0(42.6)$ & 0.47 \\
\hline Years in auto manufacturing & & & $<0.01$ \\
\hline $0-10$ years & 15 & 29 & \\
\hline $11-20$ years & 9 & 4 & \\
\hline$>20$ years & 76 & 67 & \\
\hline \multicolumn{4}{|l|}{ Medical factors } \\
\hline History of smoking & 44 & 34 & 0.04 \\
\hline Exercise regularly & 67 & 66 & 0.90 \\
\hline Diabetes & 10 & 7 & 0.30 \\
\hline Rheumatoid arthritis & 7 & 2 & 0.02 \\
\hline Osteoarthritis & 25 & 15 & 0.02 \\
\hline Forefoot pronation (by observation) & 27 & 27 & 0.97 \\
\hline Hip pain & 39 & 18 & $<0.01$ \\
\hline Knee pain & 56 & 28 & $<0.01$ \\
\hline Ankle/foot pain & 65 & 23 & $<0.01$ \\
\hline \multicolumn{4}{|l|}{ Psychosocial factors } \\
\hline Education level & & & 0.03 \\
\hline Did not complete high school & 3 & 1 & \\
\hline High school graduate & 42 & 32 & \\
\hline Some college & 36 & 34 & \\
\hline College grad or higher & 19 & 33 & \\
\hline Skill discretion (12-48) & $33.6(7.0)$ & $35.1(7.3)$ & 0.04 \\
\hline Decision authority (12-48) & $32.2(8.1)$ & $35.3(7.7)$ & $<0.01$ \\
\hline Supervisor support (4-16) & $11.5(2.2)$ & $12.5(2.2)$ & $<0.01$ \\
\hline Job dissatisfaction: $0=$ more sat., $10=$ less sat. & $2.6(2.2)$ & $1.5(1.8)$ & $<0.01$ \\
\hline Coworker support (4-16) & $11.9(1.7)$ & $12.4(1.9)$ & 0.02 \\
\hline Job insecurity (3-12) & $5.2(1.6)$ & $5.0(1.5)$ & 0.25 \\
\hline \multicolumn{4}{|l|}{ Job factors } \\
\hline Average hours worked/week & $63.8(12.3)$ & $61.1(12.4)$ & 0.07 \\
\hline \% Using shoe rotation (\%) & 43 & 50 & 0.22 \\
\hline \% Time walking or sidestepping & $25.7(12.7)$ & $24.8(12.5)$ & 0.53 \\
\hline$\%$ Time on hard surfaces & $35.4(22.6)$ & $33.3(23.6)$ & 0.40 \\
\hline$\%$ Time on carpet & $5.1(11.9)$ & $10.0(15.9)$ & $<0.01$ \\
\hline Shoe insert & 33 & 23 & 0.05 \\
\hline $\begin{array}{l}\text { Freedom to move around (for those who sit more } \\
\text { than } 50 \% \text { of the work day) } \mathrm{N}=102(\%)\end{array}$ & & & 0.01 \\
\hline Sit in a fixed position & 4 & 4 & \\
\hline Able to get up once in a while & 28 & 4 & \\
\hline Able to get up anytime & 68 & 91 & \\
\hline High durometer reading, outside of shoe & 92 & 83 & $<0.01$ \\
\hline High durometer reading, inside of shoe & 58 & 56 & 0.76 \\
\hline
\end{tabular}

${ }^{\mathrm{a}}$ Numerical variables are presented as means (standard deviation) and categorical variables are presented as percentages. 
TABLE II. Associations Between Fatigue at the End of the Day and Personal,Medical,Psychosocial, and Ergonomic Factors in Multivariate Analysis $(\mathrm{N}=365)$

\begin{tabular}{lccc} 
Risk factors & Odds ratio & P-Value & 95\% Confidence interval \\
\hline History of smoking & 1.7 & 0.05 & $1.0,2.8$ \\
History of rheumatoid arthritis & 5.8 & 0.03 & $1.2,27.9$ \\
Supervisor support & 0.9 & 0.03 & $0.8,1.0$ \\
Job dissatisfaction & 1.3 & 0.00 & $1.1,1.5$ \\
Hardness of outside of shoe (by durometer) high vs. low & 2.6 & 0.01 & $1.3,5.3$ \\
Time standing or walking on carpet (increase of 10\%) & 0.8 & 0.01 & $0.6,0.9$ \\
\hline
\end{tabular}

Pseudo $\mathrm{R}^{2}=0.13$

those with harder outsoles were more likely to report lower extremity fatigue at the end of the work day.

A logistic regression model, presented in Table II, was created with prevalence of lower extremity fatigue at the end of the day as the dependent variable and demographic, medical history and ergonomic risk factors as the independent variables. The pseudo $\mathrm{R}^{2}$ for this model was 0.13 . The odds of lower extremity fatigue increased $68 \%$ in subjects with a history of smoking. Supervisor support was protective with a $13 \%$ decreased odds for each incremental increase in support based on a 4-16 point scale. Subjects with job dissatisfaction were more likely to report lower extremity fatigue with a $26 \%$ increase in the odds of fatigue for an increase of 1 point on a $0-10$ point scale measuring job dissatisfaction. A prior diagnosis of rheumatoid arthritis increased the odds for lower extremity fatigue almost sixfold. Subjects with firmer soles on their shoes were almost 2.6 times more likely to report lower extremity fatigue at the end of the work day compared to those with subjects with shoes categorized as being "low" on the durometer reading. In those subjects who spent part of the work day on carpet, for every $10 \%$ increase in time spent standing or walking on carpet during the work day there was a $13 \%$ decrease in the odds for reporting work-related lower extremity fatigue.

A logistic regression model for lower extremity fatigue that interfered with activity outside of work is presented in Table III. As in the model for lower extremity fatigue at the end of the work day, significant predictors included rheumatoid arthritis, lower supervisor support, and greater job dissatisfaction, while standing on carpet was protective. For every 5 point increase in BMI the odds for fatigue increased $28 \%$. Subjects with a history of lower extremity osteoarthritis were 2.4 times more likely to report lower extremity fatigue severe enough to interfere with activities outside of work. Subjects who reported using additional inserts in their shoes were 2.4 times more likely to have lower extremity fatigue affecting outside activities. For the subset of participants who spent part of the work day on carpet, a $10 \%$ increase in time standing or walking on carpet reduced the odds for fatigue $34 \%$. For every additional $1 \mathrm{hr}$ worked per week, the odds for fatigue that interfered with activities outside of work increased $3 \%$. The pseudo $\mathrm{R}^{2}$ for this model was 0.18 .

\section{DISCUSSION}

While previous studies have found a wide range in the prevalence of general fatigue in the workplace our population had a high prevalence of lower extremity fatigue in comparison. This study is one of the few to focus solely on lower extremity fatigue and we report a higher prevalence compared to previously published studies. The majority of

TABLE III. Associations Between Fatigue That Interferes With Activity Outside of Work and Personal, Medical, Psychosocial, and Ergonomic Factors in Multivariate Analysis $(\mathrm{N}=341)$

\begin{tabular}{lccc} 
Risk factors & Odds ratio & P-Value & 95\% Confidence interval \\
\hline Body mass index (increase in increments of 5) & 1.3 & 0.05 & $1.0,1.6$ \\
History of rheumatoid arthritis & 4.2 & 0.03 & $1.2,15.0$ \\
History of osteoarthritis & 2.4 & 0.01 & $1.3,4.6$ \\
Supervisor support & 0.9 & 0.03 & $0.8,1.0$ \\
Job dissatisfaction & 1.3 & 0.00 & $1.1,1.4$ \\
Shoe insert & 2.4 & 0.00 & $1.4,4.1$ \\
Time standing on carpet (increase of 10\%) & 0.8 & 0.02 & $0.6,1.0$ \\
Average hours worked/week (increase in increments of $1 \mathrm{hr}$ ) & 1.03 & 0.01 & $1.1,1.8$ \\
\hline
\end{tabular}

Pseudo $\mathrm{R}^{2}=0.18$ 
subjects ( $>80 \%$ ) spent more than $50 \%$ of the work day either standing or walking and this may have contributed to the high prevalence. The timing of our study coincided with a high production period requiring consistent overtime for most workers and the higher prevalence of fatigue may be attributed in part to the longer work days and weeks of our subjects. The cohort in this study was older ( $49 \pm 9.5$ years) and had a high prevalence of osteoarthritis (22\%) which may have influenced the prevalence of lower extremity fatigue.

In his laboratory study, Kim et al. [1994] did not use subjective measurements but assessed lower extremity muscle fatigue based upon changes in surface EMG recordings. Cham and Redfern [2001] had subjects rate both overall and leg fatigue using the 10-point Borg scale. King [2002] and Orlando and King [2004] also asked subjects to rate both general fatigue and lower extremity fatigue using a 5 point Likert-scale ("not tired" to "very tired"). In the laboratory studies, subjects were asked to rate fatigue after specific periods of time spent on various work surfaces. However, this was not practical for use in the plant setting. Our questions differed from the laboratory based studies since the goal was to examine lower extremity fatigue at the work site. In an attempt to capture the extent of fatigue we asked subjects not only about fatigue at the end of the work day but how much it interferes with activities outside of work and how much fatigue persists after 1-2 days of rest from work.

We did not find a correlation between lower extremity fatigue and age. This may be attributed to a skewed distribution in the age of our study population with the majority of workers being over the age of 50 years. Given that $73 \%$ of our study population has worked in auto assembly for more than 20 years it is not unexpected that age contributes little to the predictive model for fatigue as there is likely a healthy worker effect.

Previous studies on general fatigue have found a correlation between work-related fatigue and psychosocial factors such as coworker support, job control, depression, anxiety, and emotional stress [Chen, 1986; Ricci et al., 2007; Leone et al., 2008]. While we collected psychosocial information only related to work, we did find job dissatisfaction to be associated with both lower extremity fatigue at the end of the work day and lower extremity fatigue that interferes with outside activities. Conversely, higher supervisor support was associated with a lower level of fatigue. The perception of more managerial support may lower stress resulting in fewer physical complaints and may raise the threshold for tolerating lower extremity fatigue and other discomforts associated with work. Managerial support might also mean sympathy to worker complaints which might in turn result in actions such as altered rotation schedules or work redesign.

Previous studies have examined the relationship among shoes, floor surface, time standing, physiological changes, and self-reported lower extremity fatigue with varying results. Cham and Redfern [2001] found that leg skin temperature, weight shifting, subjective discomfort, and fatigue ratings were significantly affected by floor mats. Their results showed that mats with increased elasticity and stiffness along with low energy absorption resulted in less discomfort and fatigue while a hard floor surface was associated with the highest reported levels of fatigue. Subjective measures of leg fatigue in a manufacturing plant also showed a significant increase in fatigue from standing on the hard floor compared to standing on a mat, wearing shoe inserts, or a combination of wearing shoe inserts while standing on a mat [King, 2002]. A study by Orlando and King [2004] looked at leg fatigue and differences in the type of floor surface and shoe interfaces (hard floor, mat, shoe orthoses) in assembly workers; no significant differences were found between flooring condition and subjective ratings of general fatigue, leg fatigue, and the level of discomfort experienced in various body regions. Kim et al. [1994] found that "localized" muscular fatigue on the leg may not be relieved with "anti-fatigue" mats based on results from a lab study using lower extremity surface EMG recordings as a correlate of fatigue. We also found no difference in lower extremity fatigue between those who spent more time on mats compared to those who spent more time on hard floor surfaces. We conducted a secondary analysis looking only at subjects who spent at least $10 \%$ of the work day standing or walking on mats and found that more time spent on the matting was not correlated with less fatigue. However the subset of our study population who spent part of the day on carpet did have lower odds for lower extremity fatigue, giving some credence to the current expectation that a "softer" surface is less fatigue inducing. These findings warrant further investigation into the effectiveness of floor mats to control lower extremity fatigue in people who stand or walk for the majority of the work day.

Our finding that a harder shoe sole increased the risk for lower extremity fatigue shows that it may not be enough to look at floor surface or shoe inserts alone. Rather, it is the combination of the shoe properties and floor surface that may have a greater affect on standing fatigue. This finding suggests that the type of shoe-wear, and specifically the type of heel material, could be modified in a plant setting as a primary prevention strategy.

In a plant based study of lower extremity fatigue related to standing, King [2002] reported decreased perception of fatigue with the use of shoe inserts as compared to standing on a hard surface without inserts. In contrast we found that those subjects with fatigue were more likely to use shoe inserts. We attribute this to the idea that those employees with greater fatigue are more likely to seek treatment for the condition. All employees at the site had access to medical care at a plant based clinic where free shoe inserts were available. Given that $20 \%$ of our study population sought 
treatment for lower extremity fatigue it is likely some are trying inserts as a treatment strategy.

Similar to the findings by Lewis and Wessely [1992] in a review of previous studies on the epidemiology of general fatigue, we found elevated odds for fatigue in those with rheumatoid arthritis and osteoarthritis. This is not an unexpected finding given that a primary symptom of these disorders is pain and the strong correlation between pain and fatigue. The fatigue may also be related to increased demands on the lower extremity musculature to support the dysfunctional joints.

A history of smoking has been correlated previously with exercise induced fatigue but other studies have not described smoking as a risk factor for work-related fatigue. Given the known negative effects of smoking on circulation and tissue repair it is plausible that these contribute to the presence of fatigue in the lower extremities. Reverse causation cannot be ruled out in the relationship between smoking and fatigue. It is possible that some subjects with lower extremity fatigue may continue smoking as a coping or management response to persistent lower extremity fatigue. The relationship between lower extremity fatigue and smoking warrants further investigation as a possible area of intervention to treat or prevent work-related fatigue.

Previous population-based studies have focused on general fatigue but have not evaluated work-related lower extremity fatigue. Lab based studies which include both subjective and objective measurements have been small in scale. This study is notable in that it was conducted in the workplace on a population that spent the majority of the work day standing or walking on a variety of surfaces. The detailed ergonomic assessment provides a more accurate level of information on body posture and standing surface than studies that utilize self-report for job exposures.

Limitations of this study include lack of objective measures correlated with lower extremity fatigue such as surface EMG or skin temperature. We attempted to capture not only the prevalence of lower extremity fatigue but the severity in terms of interference with activities outside of work along with treatments sought. However, direct comparison to other population-based studies is difficult due to a focus on lower extremity fatigue rather than general fatigue, and different questions used to capture fatigue data. Given the strong correlation between pain and fatigue, it is difficult to define how much pain influences fatigue (and vice versa) and to separate the prevalence of "pure" lower extremity fatigue from localized or regional pain. The pseudo $\mathrm{R}^{2}$ values for both models, $13 \%$ of the variance for lower extremity fatigue at the end of the day and $18 \%$ of the variance for lower extremity fatigue that interferes with activities outside of work, indicates that additional research is needed in this area to elucidate risk factors for lower extremity fatigue that were not evaluated in this study. Due to the cross sectional design of this study, it is unknown if there is actually reverse causation occurring with the covariates identified. For example it is unknown if the job dissatisfaction leads to increase fatigue or if experiencing fatigue regularly leads to job dissatisfaction. Unfortunately we were not given access to information on workers in the plant who chose not to participate in the study. Therefore it is unknown if there was a selection bias towards workers with lower extremity pain and/or fatigue or if there were any significant differences between participants and nonparticipants.

Prior research has described the high costs and lost productivity due to work-related fatigue [Ricci et al., 2007] but less is known about successful interventions to treat or prevent fatigue. Common interventions have been limited to shoe inserts and anti-fatigue mats. However the results of this study suggest there are other areas to be evaluated for effects on fatigue. Possible strategies to evaluate with future research are shoe recommendations, smoking cessation programs, and psychosocial aspects of work such as supervisor support.

\section{REFERENCES}

Cham R, Redfern MS. 2001. Effect of flooring on standing comfort and fatigue. Hum Factors Fall 43(3):381-391.

Chen MK. 1986. The epidemiology of self perceived fatigue among adults. Prev Med 15:74-81.

De Vries J, Michielsen HJ, Van Heck GL. 2003. Assessment of fatigue among working people: A comparison of six questionnaires. Occup Environ Med 60 (Suppl I):10-15.

Karasek R, Brisson C, Kawakami N, Houtman I, Bongers P, Amick B. 1998. The job content questionnaire (JCQ): An instrument for internationally comparative assessments of psychosocial job characteristics. J Occup Health Psychol 3(4):322-355.

Keyserling WM, Wiggermann N, Werner RA, Gell N. 2010. Interworker variability in lower body postures during assembly line work: Implications for exposure assessment. J Occup Environ Hyg 7(5):261 271.

Kim JY, Stuart-Buttle C, Marras WS. 1994. The effects of mats on back and leg fatigue. Appl Ergon 25(1):29-34.

King P. 2002. A comparison of the effects of floor mats and shoe in-soles on standing fatigue. Appl Ergon 33:477-484.

Leone S, Huibers M, Knottnerus JA, Kant I. 2008. The prognosis of burnout and prolonged fatigue in the working population: A comparison. J Occup Environ Med 50(10):1195-1202.

Lewis G, Wessely S. 1992. The epidemiology of fatigue: More questions than answers. J Epidemiol Community Health 46:92-97.

MedlinePlus. 2007. Medical Encyclopedia: Fatigue. U.S. National Library of Medicine and the National Institutes of Health. [Online] Available at www.nlm.nih.gov/medlineplus/ency/article/003088.htm (Accessed June 15, 2007).

Messing K, Tissot F, Stock S. 2008. Distal lower-extremity pain and work postures in the Quebec population. Am J Public Health 98(4):705-713.

Orlando A, King P. 2004. Relationship of demographic variables on perception of fatigue and discomfort following prolonged 
standing under various flooring conditions. J Occup Rehab 14(1): $63-76$.

Redfern MS, Chaffin DB. 1995. Influence of flooring on standing fatigue. Hum Factors 37:570-581.

Redfern MS, Cham R. 2000. The influence of flooring on standing comfort and fatigue. Am Ind Hyg Assoc J 61:700-708.
Ricci J, Chee E, Lorandeau A, Berger J. 2007. Fatigue in the U.S. workforce: Prevalence and implications for the lost productive work time. J Occup Environ Med 49(1):1-10.

Werner RA, Gell N, Hartigan A, Wiggerman N, Keyserling WM. 2010 Risk factors for plantar fasciitis among assembly plant workers. PMR 2:110-116. 